\title{
$X$-ray photoelectron and infrared spectroscopies of quartz samples of contrasting toxicity
}

\author{
Stephen M Francis*1, W Edryd Stephens ${ }^{2}$ and Neville V Richardson ${ }^{1}$
}

Address: ${ }^{1}$ School of Chemistry, University of St Andrews, Purdie Building, North Haugh, St Andrews, Fife KY16 9ST, UK and ${ }^{2}$ School of Geography \& Geosciences, University of St Andrews, Irvine Building, North Street, St Andrews, Fife KY16 9AL, UK

Email: Stephen M Francis* - smf6@st-andrews.ac.uk; W Edryd Stephens -wes@st-andrews.ac.uk; Neville V Richardson - nvr@st-andrews.ac.uk

* Corresponding author

from Joint Environment and Human Health Programme: Annual Science Day Conference and Workshop

Birmingham, UK. 24-25 February 2009

Published: 21 December 2009

Environmental Health 2009, 8(Suppl I):S4 doi:I0.II86/I476-069X-8-SI-S4

This article is available from: http://www.ehjournal.net/content/8/SI/S4

(C) 2009 Francis et al; licensee BioMed Central Ltd.

This is an open access article distributed under the terms of the Creative Commons Attribution License (http://creativecommons.org/licenses/by/2.0), which permits unrestricted use, distribution, and reproduction in any medium, provided the original work is properly cited.

\begin{abstract}
An exploratory XPS and FTIR investigation of the surfaces of bulk quartz powders widely used in toxicological studies (DQI2 and Min-U-Sil 5) was carried with the aim of correlating surface features with toxicity as reflected by indicators of biological response. Some patches of amorphous silica were identified as well as varying amounts of calcium but none of these features correlated with biological response. No evidence of widely-quoted surface silanol $(\mathrm{SiOH})$ structures was found in this investigation and the possibility that FTIR artefacts have been previously misidentified as silanol structures is discussed.
\end{abstract}

\section{Background}

Quartz dust of respirable size is associated with silicosis and other lung diseases [1] following inflammatory response to the inhaled particles [2]. The mineral surface is implicated as occlusion of the surface of quartz dusts largely eliminates the response [3] and variations in biological response are frequently attributed to variations in surface reactivity of quartz [4]. The mechanism of the effect is poorly understood and various aspects of the mineral surface have been implicated including surface free radicals, surface impurities such as $\mathrm{Fe}$, and surface silanol groups [5-7]. This study examines surface chemical characteristics of bulk quartz powders using Fourier transform infrared spectroscopy (FTIR) and X-ray photoelectron spectroscopy (XPS) with the aim of identifying structures potentially relevant to the toxic effect. Two quartz samples associated with different biological response were investi- gated. We searched for impurities and surface silanol structures with the aim of identifying any relationship to differences in known biological response for the same powders.

\section{Methods}

Samples chosen for study were Min-U-Sil 5 and DQ12, both already well characterised for particle size, surface area and biological response [8-10]. No pre-treatment or particle size separation was performed on these samples therefore all results are indicative of the as-received samples. DQ12 is regarded as particularly toxic $[8-10]$ and the spectra for this sample and the apparently less toxic MinU-Sil 5 are presented below. X-Ray photoelectron spectroscopy (XPS) data were collected on a VG ESCALAB II instrument with un-monochromated $\mathrm{Al} \mathrm{K} \alpha$ radiation. The samples were prepared in pure form by adhesion to dou- 
ble sided, electrically conducting tape. Pass energies, scan sizes and data collection times were adjusted to give the required signal to noise ratio. Analysis of the collected data was performed using the CasaXPS software. Infrared spectra were obtained using a Nicolet FTIR instrument in transmission mode on samples prepared as either a dilute solution in $\mathrm{KBr}$ and pressed into $10 \mathrm{~mm}$ diameter pellets, (a pure $\mathrm{KBr}$ disc was used as a background reference) or spread on top of a 5 bounce ZnSe ATR crystal. This ATR method is favoured as it operates on the pure samples and avoids any moisture content within the $\mathrm{KBr}$ which can cause mis-cancellation problems. In all experiments a DGTS detector was employed which is essential when performing experiments involving water adsorption (see Discussion).

\section{Results}

The XPS spectra indicated the presence of very little contamination in any sample (Figure 1). There was no evidence of any Fe-bearing species. Carbon, ubiquitous on practically all materials exposed to ambient conditions, was present on all samples analysed but in smaller quantities than might be expected given the natural origin of the samples. Detailed analysis of the O1s emission peak showed no evidence of silanol species $(\mathrm{Si}-\mathrm{OH})$ on the surface but does indicate the presence of a small amount of non-crystalline, amorphous $\mathrm{SiO}_{2}$. It was not possible to identify whether these were discrete amorphous particles or amorphous patches on crystalline particles. None of the observed surface contaminants or structures correlate with known differences in biological response.

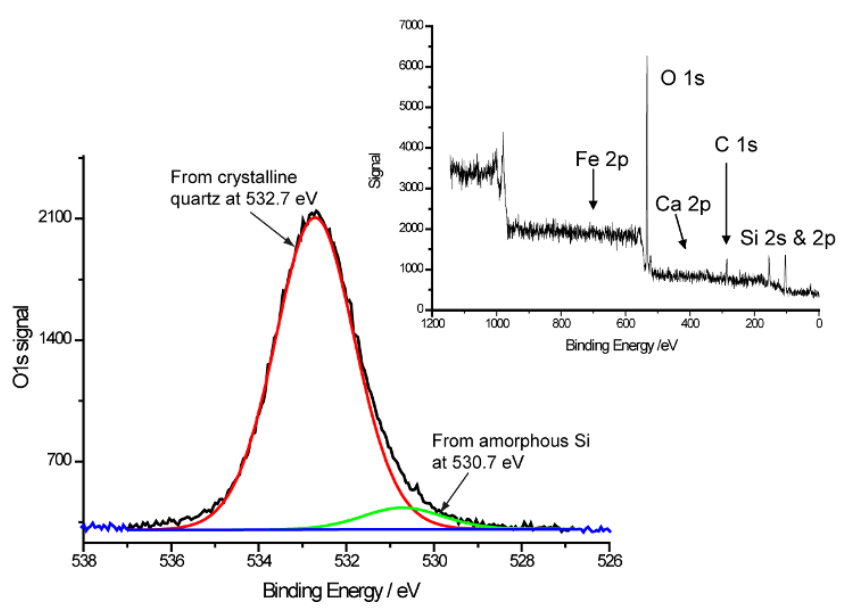

Figure I

XPS spectra of DQ 12 crystalline quartz. Oxygen Is XPS peak from DQ 12 crystalline quartz showing no evidence of a Si-OH peak at $53 \mathrm{I} .9 \mathrm{eV}$. The insert is the wide scan of the same sample indicating very little surface contamination.
The FTIR spectra of Min-U-Sil 5 obtained using the ATR method is shown in Figure 2. The insert shows that even when the O-H stretching region is displayed on an appropriate scale, no significant absorption is observed. Figure 3 shows the IR absorbance spectrum of DQ12 in the region of the bulk vibrations. This spectrum is representative of many silica samples investigated and is devoid of bands around $900 \mathrm{~cm}^{-1}$ that can be associated with hydrogen bonded Si-O-H (silanol) species.

\section{Discussion}

The work of Fubini et al [5] explicitly specifies the use of a liquid nitrogen cooled MCT detector, the same type of detector is assumed in the work of Pandurangi et al. although this is not specified [10]. An inherent problem with this type of detector is the condensation of water vapour and subsequent water ice formation on the cold detector window as a function of time. Indeed [10] shows spectra for two types of quartz samples where the O-H stretching band at $\sim 3400 \mathrm{~cm}^{-1}$ is comparable in magnitude to that of the bulk Si-O stretching bands at 1100 $\mathrm{cm}^{-1}$. This considerably exceeds what might be expected for surface $\mathrm{H}_{2} \mathrm{O}$ or $\mathrm{OH}$ species. No spectra showing the bulk bands are given in Fubini et al [5] and therefore direct comparison is not possible. Detectors of this type should be avoided when studying water/solid systems as this inherent experimental artefact will always obscure any information pertaining to the adsorption of water on the sample surface. Any data relating to this band should always be interpreted cautiously when using an MCT detector. In all studies presented here a DTGS detector was

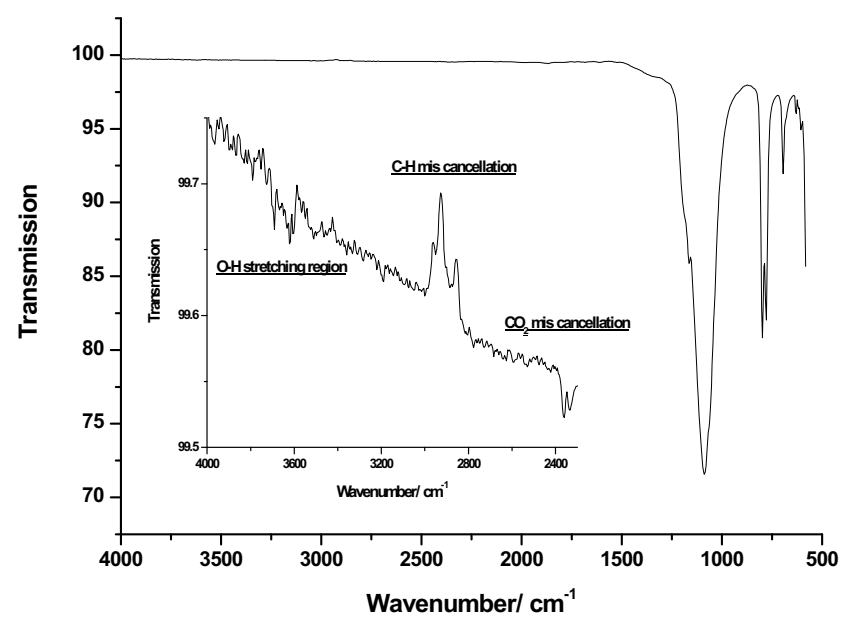

Figure 2

FTIR spectrum of Min-U-Sil 5 crystalline quartz. The IR spectrum of Min-U-Sil 5 is shown, collected using an ATR attachment combined with a DTGS detector. The O-H stretching region centred around $3500 \mathrm{~cm}^{-1}$ is shown on an appropriate scale. No evidence of a significant absorption band can be seen in this region. 


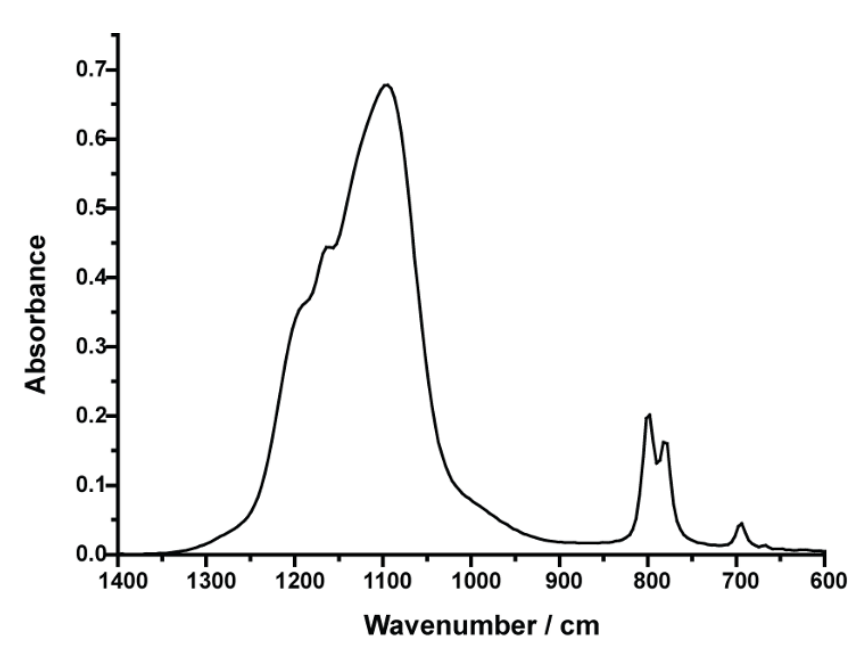

Figure 3

FTIR spectrum of DQ I 2 crystalline quartz. A small region of the FTIR spectrum of the DQI 2 quartz sample is shown. The expected silanol (hydrogen bonded $\mathrm{Si}-\mathrm{O}-\mathrm{H}$ ) band at $\sim 900 \mathrm{~cm}^{-1}$ is absent.

used, although this type of detector is not as sensitive as an MCT detector it does not suffer from this experimental disadvantage. In view of this experimental obstacle the silanol band at $\sim 900 \mathrm{~cm}^{-1}$ is taken to be a better measure of surface hydroxyl concentration.

FTIR spectra suggest that silanols are not present to any great degree on the surfaces these quartz powders, and this is in agreement with the findings from XPS. Indeed, a sample of Min-U-Sil 5 was made into a thick paste with deionised water and dried under mild conditions (24 hours at $120^{\circ} \mathrm{C}$ ) and showed no discernable difference from the untreated sample. Silanols are predicted to be widespread on hydrolysed surfaces of quartz [11] and their apparent absence is difficult to explain. Thus it was not possible to correlate silanol density with the contrasting biological responses of these two powders. Due to the nature of the toxicity tests in aqueous solution, the presence of surface silanols during the toxicity tests certainly cannot be ruled out and indeed would be expected from theoretical considerations. Pandurangi et al. [12] identified O-H bands in FTIR spectra around $3750 \mathrm{~cm}^{-1}$ and related these to their haemolysis results suggesting that the correlation was relevant to the cytotoxicity of respirable silica particles. Our work does not support this finding given the absence in our experiments of the corresponding $\mathrm{O}-\mathrm{H}$ bands at $\sim 900 \mathrm{~cm}^{-1}$ and we suggest that this relationship requires further investigation. FTIR investigations searching for surface hydroxyl species or physisorbed water is unfeasible in aqueous solution as phase modulation techniques are inappropriate for this type of system.
The XPS data does however indicate the presence of amorphous silica within the samples, this may be a fertile area for further investigation as the nature of this amorphous silica is unknown at this time. It may be distinct from or present as an integral or surface component of the crystalline quartz.

\section{Conclusion}

XPS and FTIR examination of quartz samples with varying biological response did not reveal any features of the particle surface chemistry that correlates with haemolysis or other indices of biological response. There is some evidence of amorphous silica and further investigation of this feature is necessary.

\section{Note}

The peer review of this article can be found in Additional file 1.

\section{Competing interests}

The authors declare that they have no competing interests.

\section{Authors' contributions}

SMF carried out the FTIR and XPS studies. WES, SMF and NVR conceived of the study, participated in its design and coordination, and helped to draft the manuscript. All authors read and approved the final manuscript.

\section{Additional material}

Additional file 1
Peer review.
Click here for file
[http://www.biomedcentral.com/content/supplementary/1476-
069X-8-S1-S4-S1.doc]

\section{Acknowledgements}

The authors would like to thank the Joint Environment \& Human Health Programme (NERC, EA, Defra, MOD, MRC, The Wellcome Trust, ESRC, BBSRC, EPSRC and HPA) for supporting this work through grant NE/ E009336, and to Ken Donaldson and Rodger Duffin for useful discussions and the sample of DQI2.

This article has been published as part of Environmental Health Volume 8 Supplement I, 2009: Proceedings of the Joint Environment and Human Health Programme: Annual Science Day Conference and Workshop. The full contents of the supplement are available online at http://www.ehjour nal.net/supplements/8/SI.

\section{References}

I. IARC: Silica, some silicates, coal dust and para-aramide fibrils. In IARC Monographs on the evaluation of the carcinogenic risks to humans Lyon, France; 1997:242.

2. Donaldson K, Tran CL: Inflammation caused by particles and fibers. Inhalation Toxicology 2002, I 4:5-27. 
3. Duffin R, Gilmour PS, Schins RP, Clouter A, Guy K, Brown DM, MacNee W, Borm PJ, Donaldson K, Stone V: Aluminium lactate treatment of DQ 12 quartz inhibits its ability to cause inflammation, chemokine expression, and nuclear factor-kappaB activation. Toxicology and Applied Pharmacology 200 I, I 76(I): 10-17.

4. Warheit DB, Reed KL, Sayes CM: A role for surface reactivity in TiO2 and quartz-related nanoparticle pulmonary toxicity. Nanotoxicology 2009.

5. Fubini B, Fenoglio I, Ceschino R, Ghiazza M, Martra G, Tomatis M, Borm P, Schins R, Bruch J: Relationship between the state of the surface of four commercial quartz flours and their biological activity in vitro and in vivo. International Journal of Hygiene and Environmental Health 2004, 207:89-104.

6. Fubini B, Hubbard A: Reactive oxygen species (ROS) and reactive nitrogen species (RNS) generation by silica in inflammation and fibrosis. Free Radical Biology \& Medicine 2003, 34:1507-1516.

7. Murashov V, Harper M, Demchuk E: Impact of silanol surface density on the toxicity of silica aerosols measured by erythrocyte haemolysis. Journal of occupational and environmental hygiene 2006, 3:718-723.

8. Clouter A, Brown D, Höhr D, Borm P, Donaldson K: Inflammatory effects of respirable quartz collected in workplaces versus standard DQ 2 quartz: particle surface correlates. Toxicological Sciences 200I, 63(I):90-98.

9. Bruch J, Rehn S, Rehn B, Borm PJA, Fubini B: Valuation of biological responses to different respirable quartz flours determined by a vector model. International Journal of Hygiene and Environmental Health 2004, 207:203-216.

10. Hadnagy W, Marsetz B, Idel H: Hemolytic activity of crystalline silica--separated erythrocytes versus whole blood. International journal of hygiene and environmental health 2003, 206(2): I 03- 107.

II. Bolsaitis PP, Wallace WE: The structure of silica surfaces in relation to cytotoxicity. In Silica and silica induced lung diseases CRC Press. Boca Raton, Florida; 1996:79-89.

12. Pandurangi RS, Seehra MS, Razzaboni BL, Bolsaitis P: Surface and bulk infrared modes of crystalline and amorphous silica particles: A study of the relation of surface structure to cytotoxicity of respirable silica. Environmental Health Perspectives 1990, 86:327-336.

Publish with Bio Med Central and every scientist can read your work free of charge

"BioMed Central will be the most significant development for disseminating the results of biomedical research in our lifetime. "

Sir Paul Nurse, Cancer Research UK

Your research papers will be:

- available free of charge to the entire biomedical community

- peer reviewed and published immediately upon acceptance

- cited in PubMed and archived on PubMed Central

- yours - you keep the copyright
BioMedcentral 